\title{
Spectral response of MEMS plate resonators exhibiting non-conventional vibrational modes in fluids
}

\author{
Daniel Platz ${ }^{1}$, André Gesing ${ }^{1}$ and Ulrich Schmid ${ }^{1}$ \\ ${ }^{1} T U$ Wien, Institute of Sensor and Actuator Systems, Gußhausstraße 27-29, 1040 Vienna, Austria \\ daniel.platz@tuwien.ac.at
}

\begin{abstract}
Summary:
Understanding of the fluid-structure interaction between a MEMS resonator and a surrounding fluid is key for understanding and predicting the dynamics of MEMS resonators in fluids. Here, we present a numerical method for modelling the fluid-structure interaction between a viscous fluid and vibrational eigenmodes of a plate-like MEMS resonator. The dynamics of the MEMS resonator is determined using a finite element method while the fluid flow is obtained from a boundary integral formulation. With this method we compute the spectral response of MEMS plate resonators in fluids.
\end{abstract}

Keywords: fluid sensing, MEMS resonators, fluid-structure interaction, finite elements, simulation

\section{Background, Motivation an Objective}

The characterization of fluid properties like density or viscosity is a focal area in fluid sensing. Density and viscosity sensors based on micromechanical systems (MEMS) have the potential for widespread use in various applications, like the monitoring of technical fluids like motor oil or medical diagnosis in lab-on-chip systems, due to their low-production cost and high integrability in complex sensor systems. The measurement of fluid properties requires an interaction between the MEMS sensor and the fluid environment. Such an interaction is readily established by exciting a vibrational eigenmode of a MEMS resonator. MEMS resonators often exhibit relatively simple geometries like cantilever beam structures. A typical vibrational eigenmode of a cantilever beam structure is shown in figure 1a. Advantages of beam structures are that they can be relatively simple fabricated and that their vibrational eigenmodes are readily modeled with Euler-Bernoulli beam theory. However, the quality factor of beam-like MEMS resonators is usually very low in liquids. This implies that reliable measurements of fluid properties are often difficult or even not feasible, especially in highly viscous fluids. A possible solution to this problem is the use of vibrational modes which are commonly not considered for fluid sensing. An example of such a non-conventional mode is depicted in figure $1 \mathrm{~b}$. These non-conventional vibrational modes exhibit extraordinary high quality factors in fluids [1, 2] which allows for measurements even in highly viscous fluids. Quality factors can be directly determined from the spectral response of a MEMS resonators in fluids.
However, a method for quantitatively predicting the spectra of MEMS resonators exhibiting nonconventional modes in fluids has been missing. Here, we introduce a numerical method for determining this spectral response by numerically computing the fluid-structure interaction between non-conventional vibrational modes and a surrounding fluid.

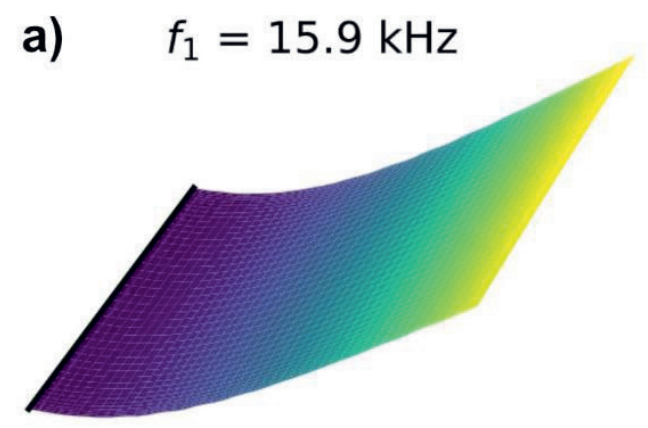

b) $f_{5}=237.4 \mathrm{kHz}$

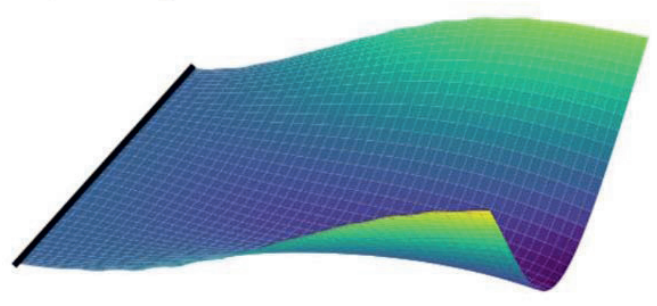

Fig. 1. Numerical simulation of vibrational eigenmodes of a cantilevered plate resonator clamped at the right edge (marked by thick black line). The mode in (a) is also observed in one-dimensional 
beam structures whereas the mode in (b) can only be found in two-dimensional structures.

\section{Description of the Method}

Two components are required for modelling the fluid-structure of non-conventional eigenmodes: the elastic dynamics the resonator and the flow of a viscous fluid around the resonator. While the elastic dynamics of conventional eigenmodes are well described by Euler-Bernoulli beam theory, the two-dimensional character of non-conventional mode requires the use of KirchhoffLove plate theory. To solve the underlying Kirchhoff-Love plate equation we use a continuous/discontinuous finite element method. This method allows for weakly imposing to the solution both the boundary conditions of a cantilevered structure and the physically motivated continuity conditions.

For MEMS resonators in the $\mathrm{kHz}$ regime the fluid flow is approximated as a Stokes flow of a viscous incompressible fluid. Moreover, due to the kinematics of non-conventional modes in slender cantilever structures, the fluid flow can be considered as approximately two-dimensional. We employ a two-dimensional stream function description of the fluid flow in planes parallel to the clamped edge of the resonator as depicted in figure 2. This description yields a boundary integral which we solve numerically.

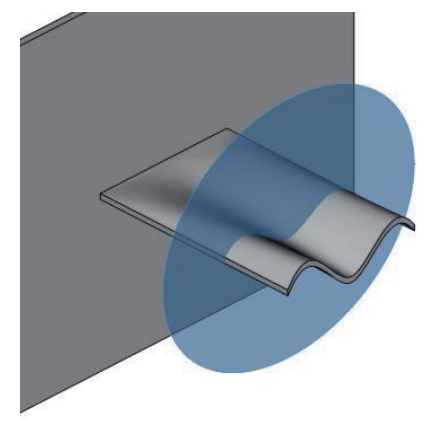

Fig. 2. Non-conventional mode of a cantilevered MEMS resonator. The blue disk indicates the plane of two-dimensional fluid flow.

Using this approach, we are able to determine the spectral response of non-conventional modes of MEMS resonators while taking into account two-dimensional plate dynamics. This has not been possible with conventional methods since most conventional methods are only applicable to one-dimensional beam modes of MEMS resonators [3].

\section{Results}

We apply the proposed method to a cantilevered plate resonator with a size of $300 \times 300 \times 5 \mu \mathrm{m}^{3}$ immersed in water. The plate is excited at one of its free corners with a force of $1 \mu \mathrm{N}$ and we compute the amplitude spectrum at this corner using the method described above. The resulting spectral is shown in figure 3 (orange line). To compare our results with conventional theory we also plot the corresponding results based on beam theory (blue line). The peak at the lowest frequency is the fundamental flexural mode which is predicted by both beam and plate theory. Both curves thus coincide. The second peak is a torsional mode not described by Euler-Bernoulli beam theory. This mode is only predicted by the plate-based theory. The peak at $100 \mathrm{kHz}$ corresponds to the second flexural mode and consequently both methods agree again with each other. The mode at $237 \mathrm{kHz}$ is the non-conventional mode shown in figure $1 \mathrm{~b}$ and its response can only be predicted with the proposed method.

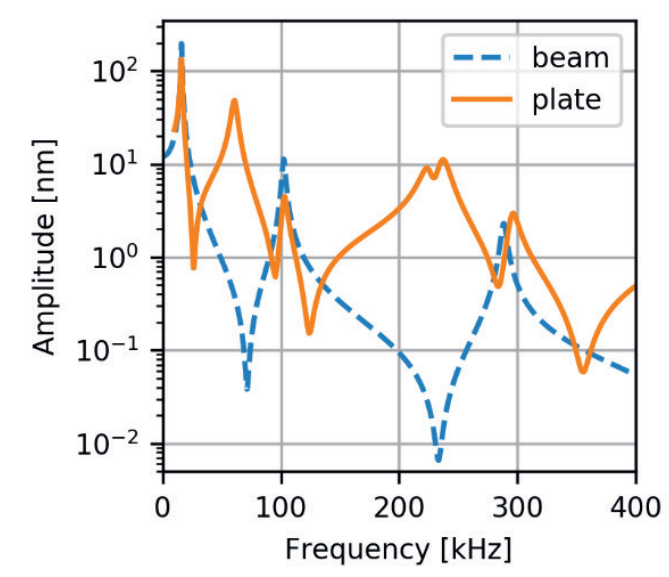

Fig. 3. Spectral response of a MEMS resonator immersed in water using beam and plate theory.

\section{Conclusion}

We present a method for modelling the fluidstructure interaction of MEMS plate resonators. With this method we are able to predict the spectral response of non-conventional modes of MEMS resonators in liquids. These spectra pave the way for quantitative measurements of fluid properties and novel designs of fluid sensors which go beyond one-dimensional beam geometries.

\section{References}

[1] Kucera, M. et al. Characterisation of multi roof tile-shaped out-of-plane vibrational modes in aluminum-nitride-actuated self-sensing micro-resonators in liquid media. Appl. Phys. Lett. 107, 053506 (2015).

[2] Pfusterschmied, G. et al. Temperature dependent performance of piezoelectric MEMS resonators for viscosity and density determination of liquids. J. Micromechanics Microengineering 25, 105014 (2015).

[3] Sader, J. E. Frequency response of cantilever beams immersed in viscous fluids with applications to the atomic force microscope. J. Appl. Phys. 84, 64-76 (1998). 